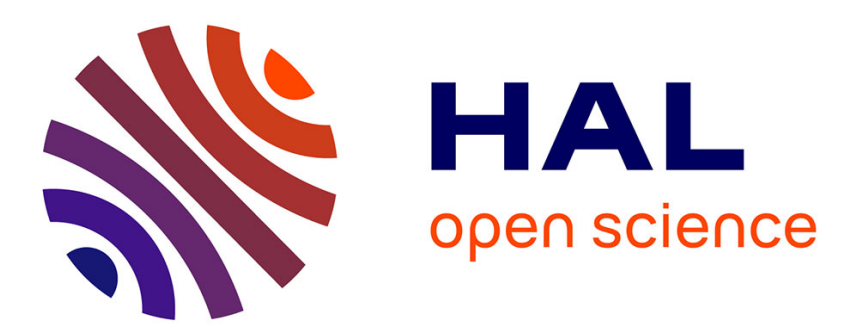

\title{
Linear instability mechanisms for coupled translational galloping
}

Angelo Luongo, Giuseppe Piccardo

\section{To cite this version:}

Angelo Luongo, Giuseppe Piccardo. Linear instability mechanisms for coupled translational galloping. Journal of Sound and Vibration, 2005, 288 (4-5), pp.1027-1047. hal-00790779

\section{HAL Id: hal-00790779 \\ https://hal.science/hal-00790779}

Submitted on 21 Feb 2013

HAL is a multi-disciplinary open access archive for the deposit and dissemination of scientific research documents, whether they are published or not. The documents may come from teaching and research institutions in France or abroad, or from public or private research centers.
L'archive ouverte pluridisciplinaire HAL, est destinée au dépôt et à la diffusion de documents scientifiques de niveau recherche, publiés ou non, émanant des établissements d'enseignement et de recherche français ou étrangers, des laboratoires publics ou privés. 


\title{
Linear instability mechanisms for coupled translational galloping
}

\author{
A. Luongo ${ }^{\mathrm{a}, *}$, G. Piccardo ${ }^{\mathrm{b}}$ \\ a Dipartimento di Ingegneria delle Strutture, delle Acque e del Terreno, University of L'Aquila, \\ 67040 Monteluco di Roio, L'Aquila, Italy

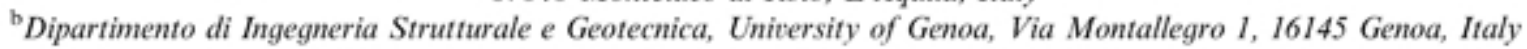

\begin{abstract}
A linearized coupled flexural two degree-of-freedom model, describing a lumped parameter system subjected to galloping, is analyzed. Through a perturbation approach, an approximated analytical solution for the eigenvalue problem is determined. Differently from the expressions existing in literature, the eigensolutions found here are valid both in quasi-resonant and non-resonant conditions. Discussing them allows depiction of the scenario of all the possible bifurcation mechanisms in the plane of the invariants of the aerodynamic damping matrix. In resonance conditions, both simple and double Hopf bifurcations are found, otherwise only simple Hopf bifurcations (eventually sequential) occur. In any case, both monomodal and bimodal galloping can take place. A closed form expression for the critical velocity is derived; it coincides with the exact solution in the resonant case and presents very good agreement with the numerical solutions in quasi-resonant conditions. The critical velocities are compared with the Den Hartog velocity and the influence of the horizontal motion is thus evaluated. Numerical examples concerning technical cases highlight the accuracy of the proposed method.
\end{abstract}

"Corresponding author. Tel.: + 39862434521 ; fax: + 390862434548 .

E mail addresses: luongo@ing.univaq.it (A. Luongo), piccardo@diseg.unige.it (G. Piccardo). 


\section{Introduction}

Galloping is a typical phenomenon of aerodynamic instability of slender structures having noncircular sections. It manifests itself with large amplitude oscillations, mainly in the crosswind direction. Tall buildings and high-rise structures, isolated structural elements, lighting pole and cables subjected to icing conditions are typical examples of structures susceptible to galloping when the wind velocity exceeds certain critical values. From a technical point of view, in order to avoid possible crisis conditions, the knowledge of the critical conditions seems particularly important even if the structural behavior could be compatible with nonlinear post-critical oscillations such as in the case of cables.

In 1932 Den Hartog introduced the first criterion concerning the critical condition for the one degree-of-freedom (dof) vertical galloping, specifying the aerodynamic loading conditions under which a horizontal conductor can gallop; the theory was developed concerning a sectional model in the quasi-static regime, which is satisfied when the characteristic fluid-dynamic time scale of the velocity fluctuations in the wake of the cylinder is much faster than the characteristic time scale of the cylinder oscillation.

The requirement of generalized critical conditions arises from the proposal of sectional models having a higher number (in general, three) of coupled dof (e.g. [1,2]). But, owing to the impossibility to obtain analytical solutions for the whole coupled model, the literature proposes results concerning two dof reduced models, as the vertical-torsion coupled galloping [3] and the coupled translational galloping [4]. It should be noted that this kind of reduced models is realistic only when the neglected mode admits a frequency far from those of the two modes taken into account. Whereas in the vertical-torsion galloping an attempt of investigating the instability trends of the dynamic behavior of a single iced conductor was proposed [5], a lack exists in the study of mechanisms governing coupled translational galloping. In this field a new criterion (called generalized Den Hartog criterion) was proposed [4] and a formula defining the onset wind velocity for the bi-dimensional coupled galloping oscillations of tower buildings was presented [6]. Both these analyses, however, are performed by supposing that the fundamental natural frequencies in the two directions are perfectly coincident. This last assumption permits simple closed-form solutions to the fourth-order characteristic equation governing the problem to be obtained. In contrast, if this hypothesis is removed, numerical methods must be employed; consequently only specific problems can be studied while an exhaustive description of all the possible critical conditions for varying system parameters is quite difficult to obtain. For this reason, the proposal of an approximate analytical method seems to be very important in order to discuss the qualitative aspects of the problem of coupled translational galloping.

In this paper a perturbation method to analytically evaluate the eigensolutions of a two dof coupled translational galloping for any frequency ratio is developed. Preliminary results were obtained in Ref. [7]. By introducing the invariants (i.e. the trace and the determinant) of the total and aerodynamic damping matrices, the eigenvalues are obtained in an expressive simple form. In particular, the conditions of incipient instability are analyzed in the plane of the aerodynamic damping matrix invariants, where each point is representative of a family of cross-sections with given aerodynamic characteristics. Unlike the literature, where single cases are analyzed, the complete scenario of all the possible bifurcation mechanisms is given in the invariants plane. Moreover, the transition from the resonant and non-resonant cases is pointed out through 
quasi-resonant conditions. The numerical applications related to examples of iced-cable sections already treated in the literature, highlight the practical interest of domains identified in the plane and permit verification of the reliability of the proposed method.

\section{Problem formulation}

Let us consider a spring-mounted damped rigid cylinder of indefinite length (i.e. a sectional model), with two translational dof, subjected to a bi-dimensional turbulent flow of horizontal uniform velocity $U$ (Fig. 1). This model is a particular case of a three dof nonlinear model developed in Refs. [1,2,8]. In these references, the alongwind and crosswind components of turbulence have been explicitly considered originating a random excitation problem. Here, instead, the influence of turbulence is restricted to a suitable modification of the values of drag and lift aerodynamic coefficients (e.g. see Ref. [9]), so that they present numerical values different from the laminar ones. In this classic approach a deterministic problem is treated and the turbulent terms did not appear explicitly in the loading terms. In the quasi-static regime (i.e. at much lower oscillation frequencies than the vortex-shedding frequency), the non-dimensional linearized equations of motion have the well-known expression (e.g. [4]):

$$
\begin{aligned}
& \mathbf{M} \ddot{\mathbf{q}}(t)+\mathbf{D} \dot{\mathbf{q}}(t)+\mathbf{K q}(t)=0, \\
& \mathbf{M}=\left[\begin{array}{ll}
1 & 0 \\
0 & 1
\end{array}\right], \quad \mathbf{D}=\left[\begin{array}{ll}
d_{11} & d_{12} \\
d_{21} & d_{22}
\end{array}\right], \quad \mathbf{K}=\left[\begin{array}{cc}
\omega^{2} & 0 \\
0 & 1
\end{array}\right], \quad \mathbf{q}(t)=\left\{\begin{array}{l}
q_{1}(t) \\
q_{2}(t)
\end{array}\right\},
\end{aligned}
$$

where $\mathbf{M} \equiv \mathbf{I}, \mathbf{D}, \mathbf{K}$ are, respectively, the mass, damping and stiffness matrices, $\mathbf{q}(t)$ is the Lagrangian displacement vector with $q_{1}(t)$ alongwind (horizontal) and $q_{2}(t)$ crosswind (vertical) displacements; dots denote dimensionless time derivatives. Constant forces, driven by the mean wind speed, are neglected as in Ref. [4], since they do not affect the oscillatory behavior of a linear system. The coefficient matrices and the dimensionless quantities involved in Eq. (1) assume the following expression:

$$
\begin{aligned}
& d_{11}=2 \omega \xi_{s}+2 \mu c_{d}, \quad d_{12}=\mu\left(c_{d}^{\prime}-c_{l}\right), \quad d_{21}=2 \mu c_{l}, \quad d_{22}=2 \xi_{s}+\mu\left(c_{d}+c_{l}^{\prime}\right), \\
& \tilde{q}_{i}=\frac{q_{i}}{b}, \quad \tilde{t}=\omega_{2} t, \quad \mu=\frac{\rho b U}{2 m \omega_{2}}, \quad \omega=\frac{\omega_{1}}{\omega_{2}},
\end{aligned}
$$

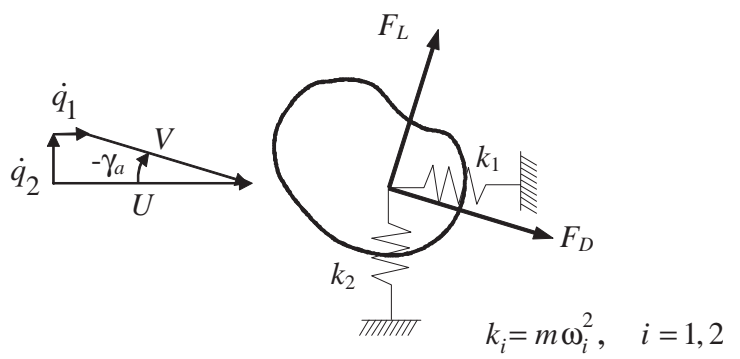

Fig. 1. Elastically supported two dof model $\left(q_{1}, q_{2}\right.$ Lagrangian displacements; $\gamma_{a} \quad$ instantaneous angle of attack; $V$ wind relative velocity; $F_{D}$ drag force; $F_{L}$ lift force). 
where $\rho$ is the fluid density, $b$ is a suitable reference length of the cylinder cross-section, $m$ is the cylinder mass per unit length, $\omega$ is the ratio between the alongwind $\left(\omega_{1}\right)$ and crosswind $\left(\omega_{2}\right)$ dimensional natural frequencies, $\xi_{s}$ is the mechanical damping ratio (assumed equal in the two directions), $c_{j}^{\prime}(j=d, l)$ are the first derivatives of drag $\left(c_{d}\right)$ and lift $\left(c_{l}\right)$ coefficients with respect to the fluctuating angle of attack, $\mu$ is the dimensionless wind velocity and $t$ is the time; finally, the tilde has been omitted in Eq. (1).

In Eqs. (2), the aeroelastic effects are accounted for by the total damping matrix $\mathbf{D}$ :

$$
\mathbf{D}=\left[\begin{array}{cc}
2 \omega \xi_{s} & 0 \\
0 & 2 \xi_{s}
\end{array}\right]+\mu\left[\begin{array}{ll}
c_{11} & c_{12} \\
c_{21} & c_{22}
\end{array}\right]=\mathbf{C}_{s}+\mu \mathbf{C}_{a},
$$

where $\mathbf{C}_{s}$ and $\mathbf{C}_{a}$ are the mechanical and aerodynamic damping matrices. Since $\mathbf{D}$ is generally full, it is responsible for the coupling between the two dof.

In order to evaluate the conditions of incipient instability, an eigenvalue problem has to be studied. The solutions to Eq. (1) are of the type $\mathbf{q}(t)=\mathbf{w} \exp (\lambda t)$, from which a homogeneous algebraic system follows:

$$
\left[\lambda^{2} \mathbf{M}+\lambda \mathbf{D}+\mathbf{K}\right] \mathbf{w}=\mathbf{0} .
$$

By setting the determinant of the matrix in Eq. (4) to zero, a fourth-order characteristic (or secular) polynomial equation, $f(\lambda ; \mu)=0$, is obtained in the unknown $\lambda=\lambda(\mu)$. When $\mathbf{D}$ is full, analytical solutions of this equation can be drawn only in particular cases, e.g. when $\omega=1$ (see Refs. $[4,6]$ ). Other significant particular conditions are the following. If a symmetric crosssection is stressed by the mean wind speed along the symmetry axis, $\mathbf{D}$ is diagonal $\left(d_{12}=d_{21}=0\right)$ and the two dof are decoupled; therefore, only a single vertical galloping can occur, since the drag coefficient $c_{d}$ and, therefore, the total alongwind damping $d_{11}$, is positive for any cross-section shape. A similar situation occurs when $d_{12}$ or $d_{21}$ vanishes; in these cases the horizontal motion is identically zero if $d_{12}=0$ or follows the leading vertical oscillations in a passive way if $d_{21}=0$. In all the previous particular cases the classic Den Hartog criterion, $d_{22}=0$, holds.

\section{Perturbation solution}

A perturbation method is applied to obtain approximate analytical expressions for the eigensolutions of system (1), valid for any frequency ratio $\omega$. A perturbation parameter $\varepsilon \ll 1$ is introduced in the equation through a suitable ordering of the coefficients. Namely, it is assumed that the coefficients of the total damping matrix $\mathbf{D}$ are small of order $\varepsilon$, i.e. $\mathbf{D}=\varepsilon \tilde{\mathbf{D}}$. This ordering is generally well founded, since the mechanical damping is small and the control parameter $\mu$ is also small for wind velocities close to the critical values of slender structures. Omitting the tilde, Eq. (4) reads:

$$
\left[\mathbf{K}+\lambda^{2} \mathbf{I}+\varepsilon \lambda \mathbf{D}\right] \mathbf{w}=\mathbf{0} .
$$

Then, the $\varepsilon$-dependent eigensolutions are expanded in MacLaurin series of $\varepsilon$ as

$$
\lambda(\varepsilon)=\lambda_{0}+\varepsilon \lambda_{1}+\varepsilon^{2} \lambda_{2}+\cdots, \quad \mathbf{w}(\varepsilon)=\mathbf{w}_{0}+\varepsilon \mathbf{w}_{1}+\varepsilon^{2} \mathbf{w}_{2}+\cdots .
$$


In order to solve Eq. (5) it is appropriate to distinguish two different cases, according to the frequency ratio $\omega$ : (a) non-resonant solution, $|\omega-1|>O(\varepsilon)$; (b) resonant and quasi-resonant solution, $|\omega-1|=O(\varepsilon)$.

\subsection{Non-resonant solution}

By substituting Eqs. (6) in Eq. (5) and equating to zero terms with the same power of $\varepsilon$, the following perturbation equations are obtained up to the $\varepsilon$-order:

$$
\begin{aligned}
& \varepsilon^{0}:\left[\mathbf{K}+\lambda_{0}^{2} \mathbf{I}\right] \mathbf{w}_{0}=0, \\
& \varepsilon:\left[\mathbf{K}+\lambda_{0}^{2} \mathbf{I}\right] \mathbf{w}_{1}=-2 \lambda_{0} \lambda_{1} \mathbf{w}_{0}-\lambda_{0} \mathbf{D} \mathbf{w}_{0} .
\end{aligned}
$$

Eq. (7a) is an eigenvalue problem, from which four eigensolutions $\left(\lambda_{0}^{(k)}, \mathbf{w}_{0}^{(k)}\right)(k=1, \ldots, 4)$ are drawn. Eq. (7b) is a singular non-homogenous problem, since $\operatorname{det}\left[\mathbf{K}+\lambda_{0}^{2} \mathbf{I}\right]=0$ for $\lambda_{0}=\lambda_{0}^{(k)}$. In order that it admits solution, the known-term must belong to the range of the operator (solvability, or compatibility, condition), i.e. it must be orthogonal to the null-space of the adjoint operator. Since the operator is self-adjoint $\left(\mathbf{K}=\mathbf{K}^{\mathrm{T}}\right)$, orthogonality to $\mathbf{w}_{0}^{(k)}$ must be enforced. From this condition, the first-order eigenvalue sensitivity $\lambda_{1}=\lambda_{1}^{(k)}$ is drawn. Then, by solving Eq. (7b), the first-order eigenvector sensitivity $\mathbf{w}_{1}=\mathbf{w}_{1}^{(k)}$ is evaluated to within an arbitrary constant, to be removed by a suitable normalization condition. The following solutions are obtained (see Appendix A for the details):

$$
\begin{aligned}
\lambda^{(1,2)}= \pm \mathrm{i} \omega-\frac{1}{2} d_{11}, & \mathbf{w}^{(1,2)}=\left(1, \mp \frac{\mathrm{i} \omega d_{21}}{1-\omega^{2}}\right)^{\mathrm{T}}, \\
\lambda^{(3,4)}= \pm \mathrm{i}-\frac{1}{2} d_{22}, & \mathbf{w}^{(3,4)}=\left(\mp \frac{\mathrm{i} d_{12}}{\omega^{2}-1}, 1\right)^{\mathrm{T}},
\end{aligned}
$$

where $\mathrm{i}$ is the imaginary unity.

\subsection{Resonant and quasi-resonant solution}

The case of nearly coincident undamped frequencies is now considered. A small detuning parameter $\sigma$ is introduced, $\omega=1+\varepsilon \sigma, \sigma=O(1)$; consequently, the stiffness matrix $\mathbf{K}$ and the total damping matrix $\mathbf{D}$ in Eq. (5) read as

$$
\mathbf{K}=\mathbf{K}_{0}+\varepsilon \mathbf{K}_{1}+O\left(\varepsilon^{2}\right), \quad \mathbf{D}=\mathbf{D}_{0}+O(\varepsilon),
$$

where

$$
\mathbf{K}_{0}=\mathbf{I}, \quad \mathbf{K}_{1}=\left[\begin{array}{cc}
2 \sigma & 0 \\
0 & 0
\end{array}\right], \quad \mathbf{D}_{0}=2 \xi_{s} \mathbf{I}+\mu \mathbf{C}_{a} .
$$

Therefore the perturbation equations (7) are modified as

$$
\begin{aligned}
& \varepsilon^{0}:\left[\mathbf{I}+\lambda_{0}^{2} \mathbf{I}\right] \mathbf{w}_{0}=0, \\
& \varepsilon:\left[\mathbf{I}+\lambda_{0}^{2} \mathbf{I}\right] \mathbf{w}_{1}=-2 \lambda_{0} \lambda_{1} \mathbf{w}_{0}-\mathbf{K}_{1} \mathbf{w}_{0}-\lambda_{0} \mathbf{D}_{0} \mathbf{w}_{0} .
\end{aligned}
$$


Eq. (11a) admits two couples of coincident eigenvalues, $\lambda_{0}^{(1,2)}=i, \lambda_{0}^{(3,4)}=-i$, whose associated eigenvectors span a plane (the whole space in this problem). However, the indeterminacy of the eigenvectors is solved by the compatibility conditions of the $\varepsilon$-order perturbation equations, from which $\lambda_{1}^{(1,2)}$ and $\lambda_{1}^{(3,4)}$ are also evaluated. The following results are obtained for the eigenvalues (see Appendix A for computations):

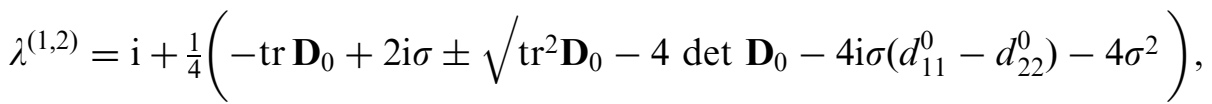

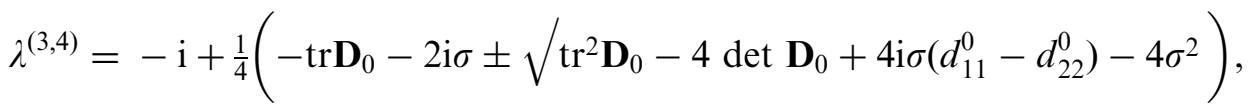

or, equivalently:

$$
\begin{aligned}
& \lambda^{(1,2)}=\mathrm{i}-\xi_{s}+\frac{1}{4}\left(-\mu \operatorname{tr} \mathbf{C}_{a}+2 \mathrm{i} \sigma \pm \sqrt{ } \mu^{2}\left(\operatorname{tr}^{2} \mathbf{C}_{a}-4 \operatorname{det} \mathbf{C}_{a}\right)-4 \mathrm{i} \sigma\left(d_{11}^{0}-d_{22}^{0}\right)-4 \sigma^{2}\right), \\
& \lambda^{(3,4)}=-\mathrm{i}-\xi_{s}+\frac{1}{4}\left(-\mu \operatorname{tr} \mathbf{C}_{a}-2 \mathrm{i} \sigma \pm \sqrt{ } \mu^{2}\left(\operatorname{tr}^{2} \mathbf{C}_{a}-4 \operatorname{det} \mathbf{C}_{a}\right)+4 \mathrm{i} \sigma\left(d_{11}^{0}-d_{22}^{0}\right)-4 \sigma^{2}\right),
\end{aligned}
$$

where the invariants of the total damping matrix $\mathbf{D}_{0}$ have been introduced:

$$
\begin{gathered}
\operatorname{tr} \mathbf{D}_{0}=d_{11}^{0}+d_{22}^{0}=4 \xi_{s}+\mu \operatorname{tr} \mathbf{C}_{a}, \\
\operatorname{det} \mathbf{D}_{0}=d_{11}^{0} d_{22}^{0}-d_{12}^{0} d_{21}^{0}=4 \xi_{s}^{2}+2 \mu \xi_{s} \operatorname{tr} \mathbf{C}_{a}+\mu^{2} \operatorname{det} \mathbf{C}_{a} .
\end{gathered}
$$

Moreover, the associated eigenvectors, corrected at the $\varepsilon^{0}$-order, are found to be

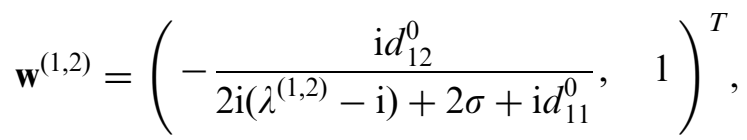

$$
\begin{aligned}
& \mathbf{w}^{(3,4)}=\left(\frac{\mathrm{i} d_{12}^{0}}{-2 \mathrm{i}\left(\lambda^{(3,4)}+\mathrm{i}\right)+2 \sigma-\mathrm{i} d_{11}^{0}}, \quad 1\right)^{T} .
\end{aligned}
$$

It should be noted that two different expressions, (12) and (13), of the resonant and quasiresonant approximate eigensolutions can be obtained using either the matrix $\mathbf{D}_{0}$ or the aerodynamic damping matrix $\mathbf{C}_{a}$, which makes explicit the role of the dimensionless wind velocity $\mu$. Eqs. (12) and (13) will be referred to as $\mathbf{D}_{0}$-representation and $\mathbf{C}_{a}$-representation of the eigenvalues, respectively.

\section{Discussion of the critical conditions}

In the previous section, approximate analytical expressions of the eigenvalues $\lambda$ were drawn for any frequency ratio $\omega$ as a function of the control parameter $\mu$. The stability critical conditions are determined by requiring $\operatorname{Re}(\lambda(\mu))=0$. In order to discuss the results, it is advisable to distinguish three main cases: (a) non-resonant conditions, $|\omega-1|>O(\varepsilon)$; (b) resonant conditions, $\omega=1$; and (c) quasi-resonant conditions, $|\omega-1|=O(\varepsilon)$. 


\subsection{Non-resonant conditions}

From Eqs. (8), just one critical condition $d_{22}=0$ follows, since $d_{11}>0$ for any cross section. Therefore, the critical wind velocity is

$$
\mu_{\mathrm{cr}} \equiv \mu_{D H}=-\frac{2 \xi_{s}}{c_{22}},
$$

which is positive if $c_{22}<0$. Eq. (16) coincides with the well-known Den Hartog criterion, $\mu=\mu_{D H}$, effective for a single vertical dof. This result highlights the fact that, in non-resonant conditions, the critical velocity is not influenced at the $\varepsilon$-order by the modal coupling. In contrast, the eigenvectors are affected by the coupling, as Eq. (8d) clarifies: if $d_{12}$ is different from zero, the horizontal motion $\left(q_{1}\right)$ is driven by the vertical unstable motion $\left(q_{2}\right)$.

\subsection{Resonant conditions}

In the case of zero detuning, $\sigma=0$ (i.e. $\omega=1$ ), Eqs. (12) and (13) simplify remarkably, since the radicands become real; therefore, they reduce respectively to

$$
\begin{aligned}
& \lambda^{(1,2)}=\mathrm{i}+\frac{1}{4}\left(-\operatorname{tr} \mathbf{D}_{0} \pm \sqrt{ } \operatorname{tr}^{2} \mathbf{D}_{0}-4 \operatorname{det} \mathbf{D}_{0}\right) \\
& \lambda^{(3,4)}=-\mathrm{i}+\frac{1}{4}\left(-\operatorname{tr} \mathbf{D}_{0} \pm \sqrt{ } \operatorname{tr}^{2} \mathbf{D}_{0}-4 \operatorname{det} \mathbf{D}_{0}\right)
\end{aligned}
$$

and

$$
\begin{aligned}
& \lambda^{(1,2)}=\mathrm{i}-\xi_{s}+\frac{\mu}{4}\left(-\operatorname{tr} \mathbf{C}_{a} \pm \sqrt{ } \operatorname{tr}^{2} \mathbf{C}_{a}-4 \operatorname{det} \mathbf{C}_{a}\right), \\
& \lambda^{(3,4)}=-\mathrm{i}-\xi_{s}+\frac{\mu}{4}\left(-\operatorname{tr} \mathbf{C}_{a} \pm \sqrt{ } \operatorname{tr}^{2} \mathbf{C}_{a}-4 \operatorname{det} \mathbf{C}_{a}\right) .
\end{aligned}
$$

In spite of the fact that Eqs. (17) and (18) are quite similar, they permit different representations on the plane of the invariants, as will soon be seen.

The $\mathbf{D}_{0}$-representation is first used (Fig. 2). It consists in evaluating on the (det $\left.\mathbf{D}_{0}, \operatorname{tr} \mathbf{D}_{0}\right)$-plane (in short, the $\mathbf{D}_{0}$-plane) the regions in which the four eigenvalues have the same character

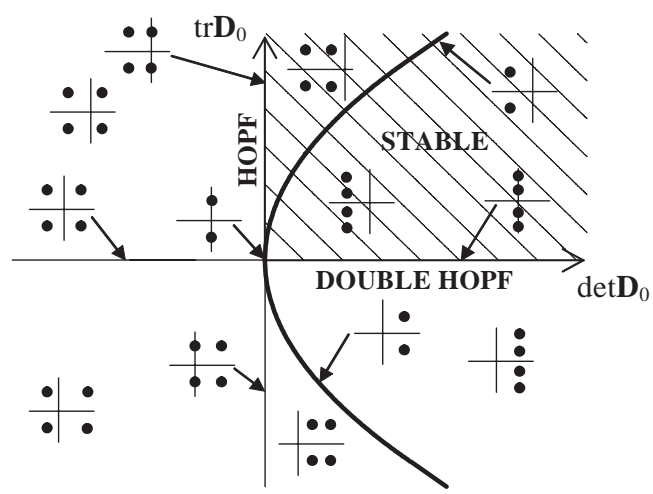

Fig. 2. Eigenvalue in the plane of the total damping matrix invariants. The parabola has equation $\operatorname{tr}^{2} \mathbf{D}_{0} \quad 4 \operatorname{det} \mathbf{D}_{0} \quad 0$. 
(real, complex or purely imaginary). In the dynamical system theory, such a plot is called a linear stability diagram. The locus of systems at incipient instability (the critical boundary), delimiting the stable region, is found by requiring that $\operatorname{Re} \lambda=0$. From Eqs. (17) the following two conditions are derived:

$$
\begin{gathered}
\operatorname{det} \mathbf{D}_{0}=0 \quad \forall \operatorname{tr} \mathbf{D}_{0}, \\
\operatorname{tr} \mathbf{D}_{0}=0 \quad \operatorname{det} \mathbf{D}_{0}>0,
\end{gathered}
$$

which represent the $\operatorname{tr} \mathbf{D}_{0}$-axis and the positive det $\mathbf{D}_{0}$-semi-axis of the $\mathbf{D}_{0}$-plane, respectively. By discussing Eqs. (17), the scenario depicted in Fig. 2 follows. The regions are bounded by the axes and by the parabola $\operatorname{tr}^{2} \mathbf{D}_{0}-4 \operatorname{det} \mathbf{D}_{0}=0$. In the first quadrant the equilibrium position is stable; along the $\operatorname{tr} \mathbf{D}_{0}$-axis a simple Hopf bifurcation occurs and along the positive $\operatorname{det} \mathbf{D}_{0}$-semi-axis a double Hopf bifurcation takes place. At the origin of the plane the double Hopf bifurcation is resonant of 1:1 type.

When $\mu=0$, the representative point has coordinates $\left(\operatorname{det} \mathbf{D}_{0}, \operatorname{tr} \mathbf{D}_{0}\right)=\left(4 \xi_{s}^{2}, 4 \xi_{s}\right)$, i.e. it lies in the first quadrant on the parabola, and the equilibrium is stable. When $\mu$ increases, $\operatorname{tr} \mathbf{D}_{0}$ and $\operatorname{det} \mathbf{D}_{0}$ vary according to Eqs. (14) and the point describes a parabola tangent to a line of angular coefficient $1 /\left(2 \xi_{s}\right)$ (Fig. 3a). If the path crosses the stability boundaries (19), then the equilibrium becomes unstable. If the damping ratio $\xi_{s}$ is fixed, the path only depends on the invariants of the aerodynamic damping matrix $\mathbf{C}_{a}$. Therefore, four cases exist according to the sign of the two $\mathbf{C}_{a^{-}}$ invariants, all described in Fig. 3a. In case (1) $\left(\operatorname{tr} \mathbf{C}_{a}>0, \operatorname{det} \mathbf{C}_{a}<0\right)$ a simple Hopf bifurcation occurs for a sufficiently large $\mu$; in case (2) $\left(\operatorname{tr} \mathbf{C}_{a}>0, \operatorname{det} \mathbf{C}_{a} \geqslant 0\right)$ the stability boundaries are not crossed and the equilibrium remains stable for any $\mu$; in case (3) $\left(\operatorname{tr} \mathbf{C}_{a}<0\right.$, $\left.\operatorname{det} \mathbf{C}_{a} \leqslant 0\right)$, simple Hopf bifurcations again occur; in case (4) $\left(\operatorname{tr} \mathbf{C}_{a}<0, \operatorname{det} \mathbf{C}_{a}>0\right)$ two sub-cases are possible: for small $\operatorname{det} \mathbf{C}_{a}$ (case 4a) two successive simple Hopf bifurcations take place, while for large $\operatorname{det} \mathbf{C}_{a}$ (case $4 \mathrm{~b}$ ) a double Hopf bifurcation manifests itself. In Fig. $3 \mathrm{~b}$ the eigenvalue paths corresponding to the four cases previously described are also shown. It is seen that, since Re $\lambda$ is a monotonic function of $\mu$, it is not possible for the system to regain stability once it has lost it.

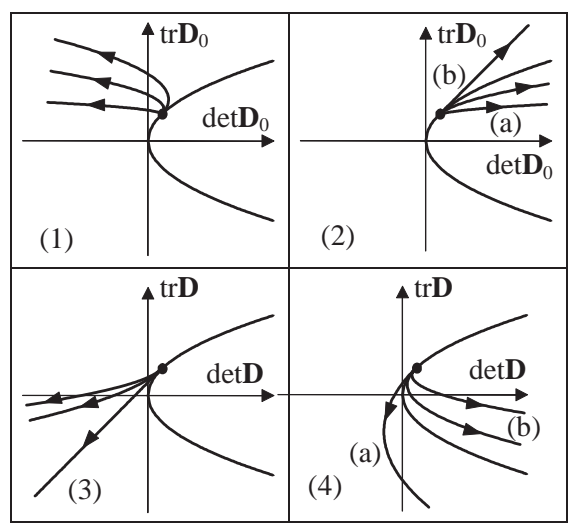

(a)

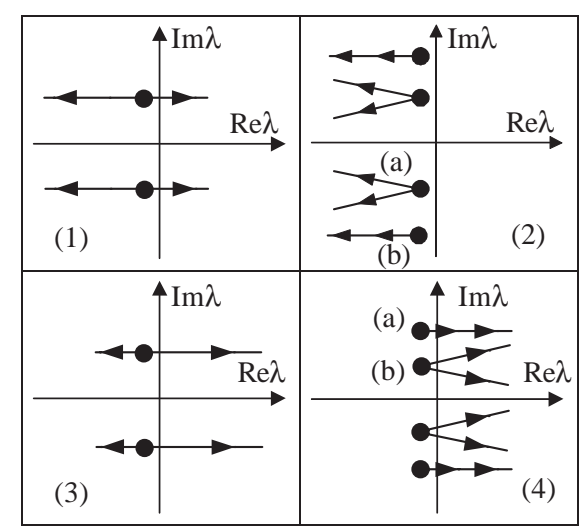

(b)

Fig. 3. (a) Invariant and (b) eigenvalue paths for increasing wind velocity; (1) $\operatorname{tr} \mathbf{C}_{a}>0$, $\operatorname{det} \mathbf{C}_{a}<0$; (2) $\operatorname{tr} \mathbf{C}_{a}>0, \operatorname{det} \mathbf{C}_{a} \geqslant 0$; (3) $\operatorname{tr} \mathbf{C}_{a}<0, \operatorname{det} \mathbf{C}_{a} \leqslant 0$; (4) $\operatorname{tr} \mathbf{C}_{a}<0, \operatorname{det} \mathbf{C}_{a}>0$; (2a,4a) small $\operatorname{det} \mathbf{C}_{a}$, (2b,4b) large $\operatorname{det} \mathbf{C}_{a}$. 


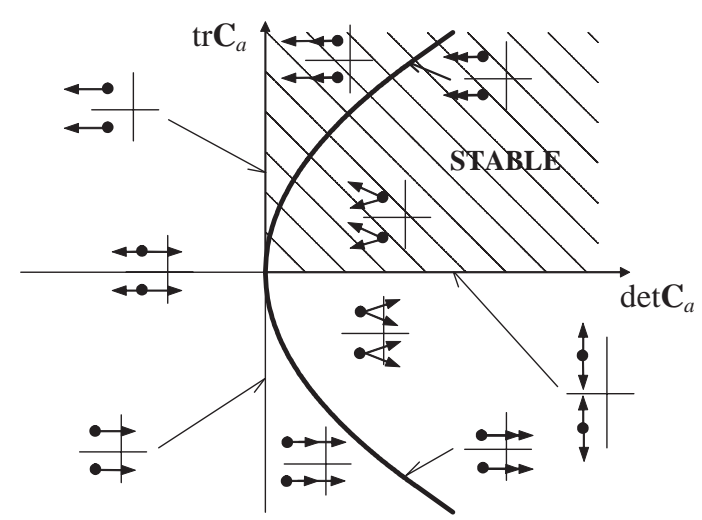

Fig. 4. Eigenvalue paths for increasing wind velocities in the plane of the aerodynamic damping matrix invariants; resonant case. The parabola has equation $\operatorname{tr}^{2} \mathbf{C}_{a} \quad 4 \operatorname{det} \mathbf{C}_{a} \quad 0$.

The $\mathbf{C}_{a}$-representation (18) is then illustrated (Fig. 4). Each point of the $\mathbf{C}_{a}$-plane is representative of a family of cross-sections with given aerodynamic characteristics. However, a physical limit exists, due to the fact that $c_{11}$ cannot assume negative or zero values; therefore $\operatorname{tr} \mathbf{C}_{a}>c_{22}$ is a lower bound for the plane. When $\mu$ is growing and it reaches a critical value, a specific bifurcation mechanism associated with that point manifests itself. By discussing Eqs. (18) for $\mu$ increasing from zero, the eigenvalue paths that characterize each region of the $\mathbf{C}_{a}$-plane are determined as illustrated in Fig. 4. It is seen that the first quadrant, including the positive semiaxes, is stable for any $\mu$; in the second and third quadrant a simple Hopf bifurcation occurs at a critical value; in the fourth quadrant two types of bifurcations exist: inside the parabola $\operatorname{tr}^{2} \mathbf{C}_{a}-4 \operatorname{det} \mathbf{C}_{a}=0$, on which the radicand in Eqs. (18) vanishes, a double Hopf bifurcation occurs, and outside the parabola two successive simple Hopf bifurcations take place. The mechanisms are obviously the same illustrated in Fig. 3b. On the parabola, a double Hopf bifurcation occurs with coincident critical eigenvalues (1:1 resonant double Hopf bifurcation). Since the geometric multiplicity of the critical eigenvalue is less than its algebraic multiplicity, the system is defective at the criticality. Inside the parabola, a non-resonant (non-defective) double Hopf bifurcation manifests itself, with the possible origin of quasi-periodic solutions (like those commented in Ref. [4]). However, a nonlinear analysis of such a bifurcation performed on nonlinear models of single taut strings [10] proved that the steady motion in the postcritical range is periodic.

From Eqs. (18), or equivalently from Eqs. (19), the lowest critical wind velocity is evaluated as a function of the $\mathbf{C}_{a}$-invariants. It is found that

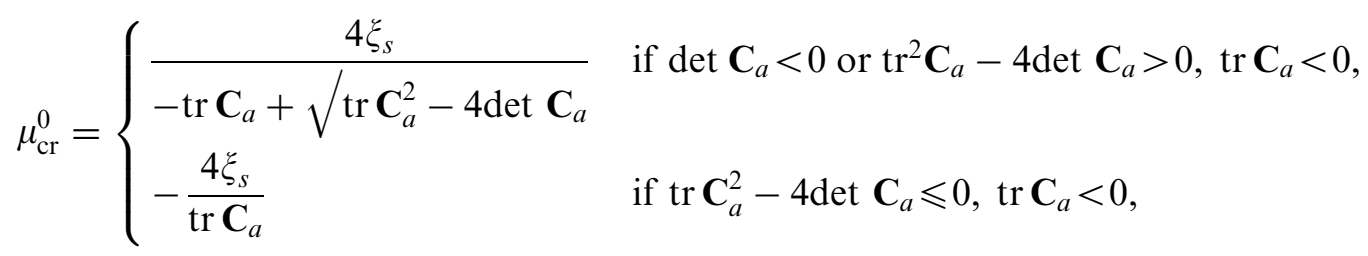

where the apex 0 on $\mu_{\mathrm{cr}}$ denotes the resonant value. Therefore, Eq. (20a) is valid in the second and third quadrant and outside the parabola in the fourth quadrant of the $\mathbf{C}_{a}$-plane; Eq. (20b) is valid 

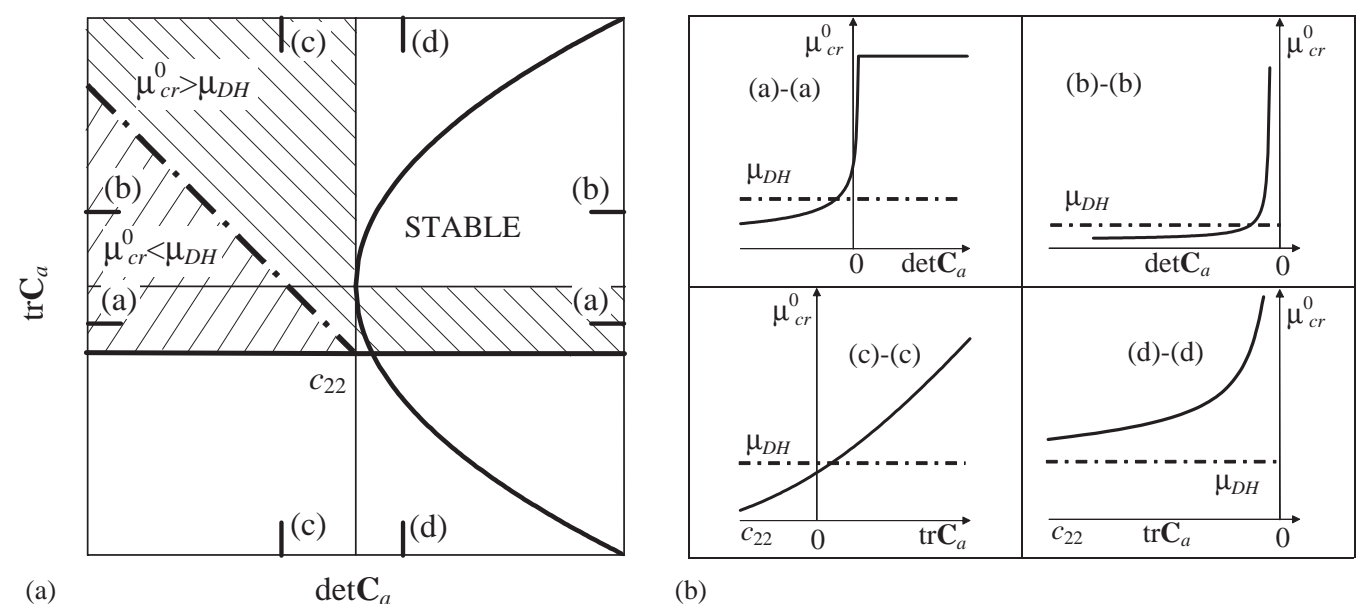

(b)

Fig. 5. Critical wind velocity in the resonant case compared with the Den Hartog value; (a) domains of the $\mathbf{C}_{a}$ plane and selected paths, (b) $\mu_{\mathrm{cr}}^{0}$ vs. the invariants along the paths.

in the fourth quadrant inside the parabola. These expressions coincide with the exact solution, deduced in Ref. [4]. Therefore, the perturbation solution gives the exact real part of the eigenvalues whereas it furnishes an approximate imaginary part.

It is interesting to compare the $\mu_{\mathrm{cr}}^{0}$ critical values (20) with the $\mu_{D H}$ Den Hartog value (16). The case $c_{22}<0$, necessary for the existence of a positive Den Hartog velocity, is considered first. By setting $\mu_{\mathrm{cr}}^{0}=\mu_{D H}$, the following relationship is found:

$$
\operatorname{tr} \mathbf{C}_{a}=\frac{\operatorname{det} \mathbf{C}_{a}}{c_{22}}+c_{22}
$$

that represents a line in the ( $\operatorname{det} \mathbf{C}_{a}, \operatorname{tr} \mathbf{C}_{a}$ )-plane (dash dotted straight line in Fig. 5a, where only the physically meaningful part of the plane has been considered). Four significant paths were selected in Fig. 5a and the relevant $\mu_{\mathrm{cr}}^{0}$ values shown in Fig. 5b. Along path a a, going from left to right, the critical velocity is initially lower than the Den Hartog velocity; after the intersection with the straight line (21), the velocity strongly increases until it reaches the constant value (20b) inside the parabola. The pattern is quite similar along path $\mathrm{b} b$; however, here $\mu_{\mathrm{cr}}^{0}$ tends to infinity when the trace-axis is approached. Along path c c, going from the bottom upwards, the critical velocity monotonically grows from a value lower than Den Hartog velocity. Finally, going from the bottom upwards along path $\mathrm{d} d$, the critical velocity is always greater than Den Hartog value and it tends to infinity when trace approaches zero.

Now, it is important to analyze the case $c_{22}>0$. According to the Den Hartog criterion the equilibrium is always stable. However, since $\operatorname{tr} \mathbf{C}_{a}>0$, instability occurs in the second quadrant of the $\mathbf{C}_{a}$-plane, as a result of the coupling between horizontal and vertical displacements.

\subsection{Quasi-resonant conditions}

The case of non-zero detuning is now considered. By zeroing the real part of the eigenvalues (12) and performing some algebraic manipulations, the following bifurcation equation is 
obtained:

$$
\operatorname{tr} \mathbf{D}_{0} \sqrt{ } \operatorname{det} \mathbf{D}_{0}+\sigma^{2}= \pm \mu\left|\sigma\left(c_{11}-c_{22}\right)\right|
$$

under the condition det $\mathbf{D}_{0}+\sigma^{2}>0$ and where the double sign is relevant to the two couples of the eigenvalues. As particular cases, Eqs. (19) are recovered when $\sigma \rightarrow 0$ and the Den Hartog criterion is obtained when $\sigma \rightarrow \infty$. It is observed that the stability of the quasi-resonant system is governed by four parameters: the detuning $\sigma$, the difference $\left(\begin{array}{lll}c_{11} & c_{22}\end{array}\right)$ and the two invariants $\operatorname{tr} \mathbf{D}_{0}$ and $\operatorname{det} \mathbf{D}_{0}$. Therefore, the problem is much more complex than in the resonant case where only the invariants are present. In particular, the $\mathbf{D}_{0}$-representation used in Figs. 2 and 3 loses its usefulness, since the stability boundaries of the $\mathbf{D}_{0}$-plane now move with the control parameter $\mu$. Hence, in order to investigate the bifurcation mechanisms, use is made of the $\mathbf{C}_{a}$-representation. Moreover, since expressions (13) of the eigenvalues are very involved, the bifurcation equation (22) is directly discussed.

Using Eqs. (14) and defining

$$
\begin{aligned}
f(\mu) & :=4 \xi_{s}+\mu \operatorname{tr} \mathbf{C}_{a}, \quad g(\mu):=\frac{\mu}{\sqrt{ } p(\mu)}, \\
p(\mu) & :=\mu^{2} \operatorname{det} \mathbf{C}_{a}+2 \mu \xi_{s} \operatorname{tr} \mathbf{C}_{a}+\left(4 \xi_{s}^{2}+\sigma^{2}\right), \quad k:=\left|\sigma\left(c_{11}-c_{22}\right)\right|
\end{aligned}
$$

the bifurcation equation (22) reads

$$
f(\mu)= \pm k g(\mu)
$$

The roots $\mu=\mu_{\mathrm{cr}}$ of Eq. (22) are therefore the abscissas of the intersections of the graphs of the two functions $f(\mu)$ and $\pm k g(\mu)$. The graph of $f(\mu)$ is a straight line, whose angular coefficient is $\operatorname{tr} \mathbf{C}_{a}$; the graph of $g(\mu)$ depends on both the invariants of $\mathbf{C}_{a}$. However, it is shown in Appendix B that $g(\mu)$ assumes only four qualitatively different patterns, essentially depending on the roots of the polynomial $p(\mu)$, each in a region of the $\mathbf{C}_{a}$-plane. The regions are bounded by the axes and by the parabola $\operatorname{tr}^{2} \mathbf{C}_{a}-\left(4+\sigma^{2} / \xi_{s}^{2}\right)$ det $\mathbf{C}_{a}=0$ (see Fig. 13 in Appendix B); the corresponding graphs of $g(\mu)$, together with the graphs of $f(\mu)$, are plotted in Fig. 6. Cases different from those shown cannot occur, so the figure is exhaustive.

The following conclusions are drawn from Fig. 6. In domain A (first quadrant of the $\mathbf{C}_{a}$-plane) two different situations exist: no bifurcation, when $k$ is sufficiently small, or one single Hopf bifurcation with successive stability regain (transient instability), when $k$ is sufficiently large (the same branch is crossed twice). It can be proved that, if $c_{22}>0$, no bifurcation exists in domain $\mathrm{A}$. In domain B (fourth quadrant, region internal to the parabola) two successive Hopf bifurcations take place (two different branches are crossed). A similar circumstance is verified in domain $\mathrm{C}$ (fourth quadrant, region external to the parabola) with two sequential bifurcations. Finally, in domain D (second and third quadrants) only a single bifurcation occurs.

Fig. 7 summarizes all the possible critical conditions exhibited by the quasi-resonant system. It is interesting to compare it with Fig. 4, which concerns the resonant case. It is seen that the first quadrant, which is stable in the resonant case, can now become unstable if $c_{22}<0$, although the instability is of transient type. For a given point of coordinates $\left(\operatorname{det} \mathbf{C}_{a}, \operatorname{tr} \mathbf{C}_{a}\right)$ the stability or instability depends on the two remaining parameters, $\sigma$ and $\left(\begin{array}{lll}c_{11} & c_{22}\end{array}\right)$. The curve $\mathscr{C}$ dividing the first quadrant into two zones has a shape like that in Fig. 7; it has been determined, for fixed $\sigma$ and $\left(\begin{array}{ll}c_{11} & c_{22}\end{array}\right)$, as locus of points $\left(\operatorname{det} \mathbf{C}_{a}, \operatorname{tr} \mathbf{C}_{a}\right)$ for which $f(\mu)$ is tangent to $+k g(\mu)$ (see Fig. 6, 


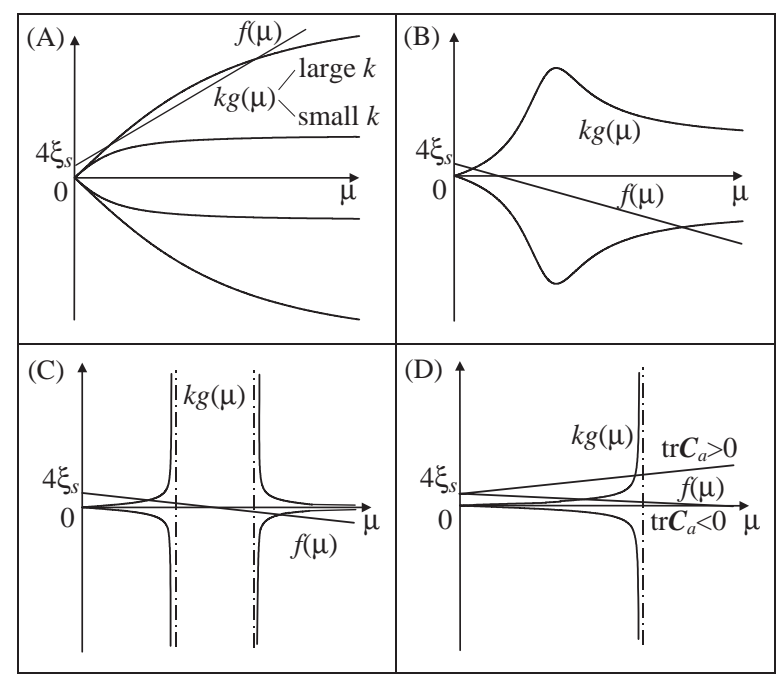

Fig. 6. Graphic solution of the bifurcation equation (24) in the quasi resonant case. Domains A,B,C and D as in Fig. 14 (Appendix B).

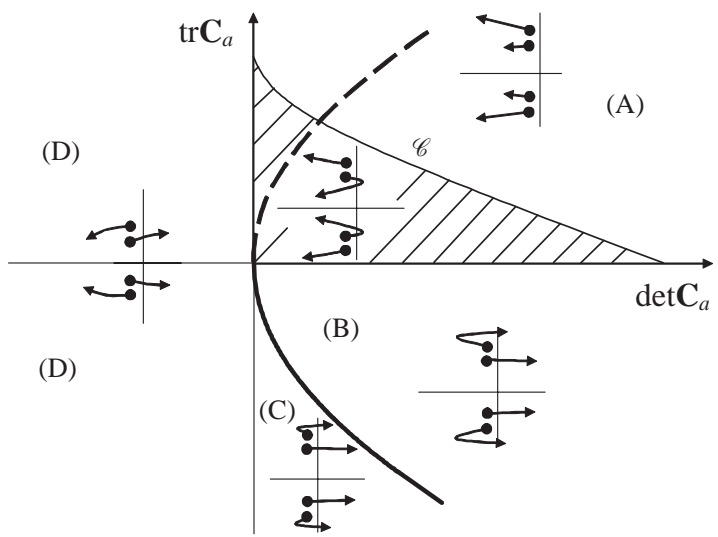

Fig. 7. Eigenvalue paths for increasing wind velocities in the plane of the aerodynamic damping matrix invariants; quasi resonant case. The parabola has equation $\operatorname{tr}^{2} \mathbf{C}_{a} \quad\left(4+\sigma^{2} / \xi_{s}^{2}\right) \operatorname{det} \mathbf{C}_{a} \quad 0$; curve $\mathscr{C}$ exists only if $c_{22}<0$.

domain A). If $\operatorname{det} \mathbf{C}_{a}$ and $\operatorname{tr} \mathbf{C}_{a}$ are slightly decreased from the value they assume on $\mathscr{C}$, then $f(\mu)$ crosses $+k g(\mu)$ (see Fig. 6). Therefore, the bounded region of the first quadrant is unstable while the unbounded region is stable. If $|\sigma|$ and $\left|c_{11} c_{22}\right|$ are increased, the unstable region also increases. If $c_{22}>0 \mathscr{C}$ does not have real values and no unstable region exists in the first quadrant. Passing to consider the other regions of the $\mathbf{C}_{a}$-plane, the qualitative behavior of the system in the second and third quadrant is substantially unchanged with respect to the resonant case. In contrast, in the fourth quadrant, the detuning inhibits the double Hopf bifurcation, which is replaced by two successive bifurcations. 


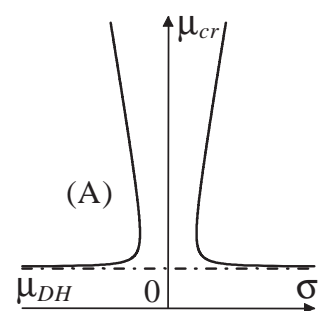

(a)

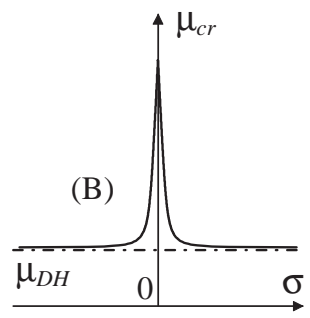

$\sigma$

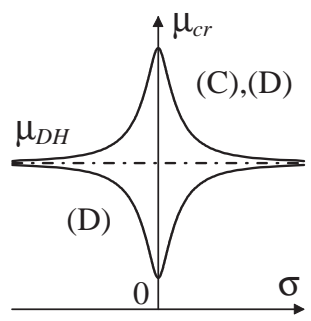

$\sigma$

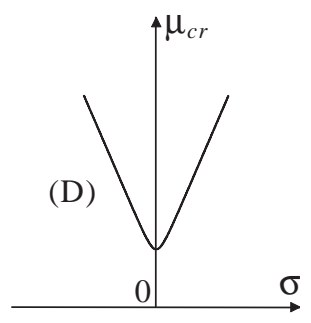

(b)

Fig. 8. Critical wind velocity vs. detuning for the domains in Fig. 7; (a) $c_{22}<0$, (b) $c_{22}>0$.

The influence of the detuning on the critical velocity is then analyzed. Eq. (22) cannot be solved in a simple closed form to furnish $\mu_{\mathrm{cr}}$ as a function of $\sigma$; however it can easily be solved to obtain $\sigma$ as a function of $\mu_{\mathrm{cr}}$. Qualitative plots of the function are represented in Fig. 8a for $c_{22}<0$ and in Fig. $8 \mathrm{~b}$ for $c_{22}>0$, as regards different domains of the $\mathbf{C}_{a}$-plane. Sections with $c_{22}<0$ are first considered. It is seen that, in all cases, $\mu_{\mathrm{cr}}$ is above or below the Den Hartog value for any $\sigma$; in addition, when $|\sigma| \rightarrow \infty, \mu_{\mathrm{cr}} \rightarrow \mu_{D H}$. In particular, $\mu_{\mathrm{cr}}>\mu_{D H} \forall \sigma$ in the domains A, B and C, whereas $\mu_{\mathrm{cr}}>\mu_{D H} \forall \sigma$ or $\mu_{\mathrm{cr}}<\mu_{D H} \forall \sigma$ in the domain D; in this latter case the resonant $\mu_{\mathrm{cr}}^{0}$ value solves the ambiguity. As regards domain A no instability occurs when $\sigma=0$, but it appears when the detuning is sufficiently large, according to the behavior described in Fig. 7. Fig. 8a also highlights the fact that the regain of stability is effective only if $\sigma$ is sufficiently small, otherwise it occurs for very large values of the wind velocity. In domain B the graph of $\mu_{\mathrm{cr}}(\sigma)$ presents a cusp at $\sigma=0$, whereas in domains $\mathrm{C}$ and $\mathrm{D}$ it has zero slope. Therefore sensitivity of $\mu_{\mathrm{cr}}$ at $\sigma=0$ is larger in domain B. However, except for a very small neighborhood of $\sigma=0$, the patterns of all these curves are similar. Finally, if the case $c_{22}>0$ is considered (Fig. 8d), instability can only occur in domain D. The Den Hartog velocity is now negative, i.e. the system is stable far from the resonance; consequently, when $|\sigma| \rightarrow \infty$ the critical velocity tends to infinity too.

\section{Numerical examples}

A numerical analysis is performed for sample sections having significant aerodynamic coefficients. The critical wind velocity is first evaluated as a function of the detuning parameter; then, some aspects of the galloping motion are studied.

\subsection{Critical wind velocity}

The accuracy of the perturbation solutions is checked by comparing them with the exact (numerical) solutions of the eigenvalue problem (4). Two different families of iced cable crosssections, NDT and NB, already discussed in Ref. [4] with regard to the resonant case, are examined and the results are reinterpreted in the light of the previous discussion. In each family a section with given aerodynamic characteristics $c_{d}, c_{l}, c_{d}^{\prime}$ and $c_{l}^{\prime}$ is taken; then, a single parameter is varied $\left(c_{d}\right.$ in the NDT family and $c_{l}$ in the NB family). Correspondingly, the representative point 
of the family's element, describes a path in the ( $\operatorname{det} \mathbf{C}_{a}, \operatorname{tr} \mathbf{C}_{a}$ )-plane (see Fig. 9), thus exploring the regions characterized by different qualitative behaviors (remember Fig. 7). It should be noted that, according to Fig. 7, the parabola on invariant plane depends on $\sigma$; owing to the small value of damping, here assumed equal to $0.7 \%$, the parabola rapidly becomes flatter for increasing $\sigma$.

The NDT family is considered first. Initially the representative point is located in domain A $\left(\operatorname{tr} \mathbf{C}_{a}>0\right.$, det $\mathbf{C}_{a}>0$ ); then it moves to domain B. For significant values of $c_{d}$ (points $\mathrm{P}_{1}, \mathrm{P}_{2}, \mathrm{P}_{3}$ in Fig. 9) the graphs of the critical velocity vs. the detuning are plotted in Fig. 10 and compared with the exact numerical values. They are of the types illustrated in Figs. 8a (first and second graph) and, in addition, show the transition between them. When $c_{d}=1.01$ (point $\mathrm{P}_{1}$ in Fig. 9) the system is subjected to transient instability for sufficiently large values of the detuning $|\sigma|$ (Fig. 10a); therefore the unstable region $\mathrm{U}$ of the $\left(\mu_{\mathrm{cr}}, \sigma\right)$-plane has lower and upper bounds. It is seen that the accuracy of the perturbation solution decreases with $|\sigma|$ with regard to the second bifurcation; this

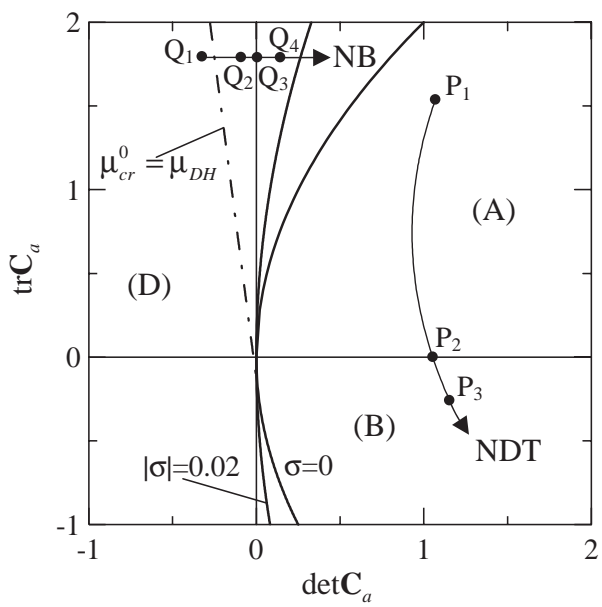

Fig. 9. Representative points of the NDT and NB family's element (the line $\mu_{\mathrm{cr}}^{0} \quad \mu_{D H}$ refers to NB family).
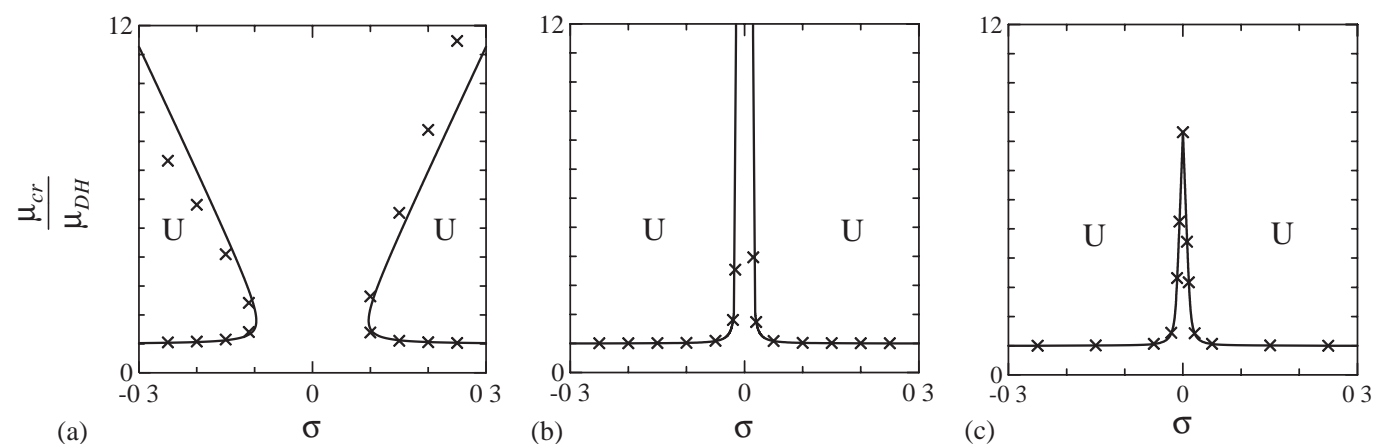

Fig. 10. Critical wind velocity vs. detuning for the NDT cross section family at points $\mathrm{P}_{1} \mathrm{P}_{3}$ of Fig. 9: $c_{l}$ 1.01, $\begin{array}{llllll}c_{d}^{\prime} & 0, c_{l}^{\prime} & 1.49\end{array}$; (a) $c_{d} \quad 1.01$, (b) $c_{d} \quad c_{l}^{\prime} / 3$, (c) $c_{d} \quad 0.41 ; \mathrm{U} \quad$ unstable regions; $\times \quad$ numerical values. 
is due to a partial ordering violation occurring at high wind velocities at which stability is regained. When $c_{d}=-c_{l}^{\prime} / 3, \operatorname{tr} \mathbf{C}_{a}=0$ and the representative point crosses the boundary between the A- and B-domains (point $\mathrm{P}_{2}$ in Fig. 9); the associated $\mu_{\text {cr }}$ vs. $\sigma$ curve is shown in Fig. 10b. The curve has two vertical asymptotes at $\sigma^{*}= \pm 4 \xi_{s} \sqrt{ } \operatorname{det} \mathbf{C}_{a} /\left(c_{11}-c_{22}\right)$, so that the section is stable for $|\sigma|<\left|\sigma^{*}\right|$ and unstable for $|\sigma|>\left|\sigma^{*}\right|$, without stability regain, i.e. the unstable regions do not have upper bounds. When $c_{d}$ is further decreased $\left(c_{d}=0.41\right.$, point $\mathrm{P}_{3}$ in Fig. 9), $\operatorname{tr} \mathbf{C}_{a}$ becomes negative and the point enters the B-domain. The relevant $\mu_{\text {cr }}$ vs. $\sigma$ curve, plotted in Fig. 10c, shows that the two unstable regions merge, so that instability occurs even at the resonance, although at a velocity greater than the Den Hartog value.

The NB family is now considered. Initially the representative point is located in domain D ( $\operatorname{tr} \mathbf{C}_{a}>0, \operatorname{det} \mathbf{C}_{a}<0$ ), in the region $\mu_{\mathrm{cr}}^{0}<\mu_{D H}$; then it moves to domain A. The $\mu_{\mathrm{cr}}$ vs. $\sigma$ curves are plotted in Fig. 11, and are of the types shown in Figs. 8a (first and third graph). The curve associated with the starting value $c_{l}=-0.42$ (point $\mathrm{Q}_{1}$ in Fig. 9) is represented in Fig. 11a. By slightly decreasing the lift coefficient $\left(c_{l}=-0.49\right)$ the line $\mu_{\mathrm{cr}}^{0}=\mu_{D H}$ is crossed (this situation corresponds to the vanishing of the $d_{21}^{0}$ coefficient, while in Ref. [4] it was reached setting $c_{l}=0$, for which $d_{21}^{0}$ instead vanishes). For smaller $c_{l}$ (point $\mathrm{Q}_{2}$ in Fig. 9) the critical velocity becomes larger than the Den Hartog value and the curve assumes the shape of Fig. 11b. By further decreasing $c_{l}$, the boundary $\operatorname{det} \mathbf{C}_{a}=0$ is first crossed (point $\mathrm{Q}_{3}$ in Fig. 9) and then domain $\mathrm{A}$ is entered (point $\mathrm{Q}_{4}$ in Fig. 9), for which the curves are shown in Fig. 11c. Also in this case the ordering violation entails quantitative errors.

As a numerical example of the $\mathscr{C}$-curves delimiting the (transient) unstable part of domain A (see Fig. 7), the curves plotted in Fig. 12 have been determined for $c_{11}-c_{22}=2.05$, i.e. for the NB family. It is seen that, due to the high value of $\operatorname{tr} \mathbf{C}_{a}$ for the NB family, the section is stable even for moderately large $|\sigma|$. The higher $\operatorname{det} \mathbf{C}_{a}$, the higher the value of $|\sigma|$ at which transient instability manifests itself, according to Fig. 11c.

Concerning other cross-sections considered in the literature, most of them have $\operatorname{tr} \mathbf{C}_{a}$ positive and are sited in domains $\mathrm{A}$ and $\mathrm{D}$. Some examples belonging to domain $\mathrm{B}$ also appear, as has been seen. Cases concerning domain $\mathrm{C}$ instead are not very likely, also in consideration of the lower limit of the trace, $\operatorname{tr} \mathbf{C}_{a}>c_{22}$. For example, a classic technical case concerns the 2-D coupled
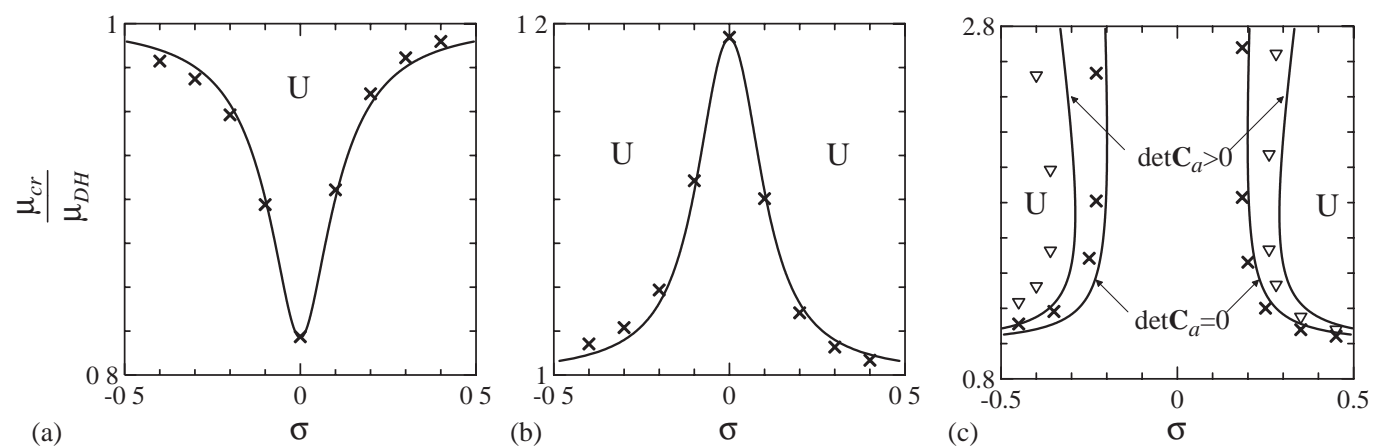

Fig. 11. Critical wind velocity vs. detuning for the NB cross section family at points $\mathrm{Q}_{1} \mathrm{Q}_{4}$ of Fig. 9: $c_{d}$ 0.96,

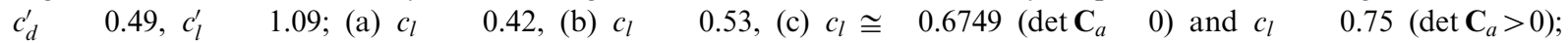
$\mathrm{U}$ unstable regions; $\times, \nabla$ numerical values. 


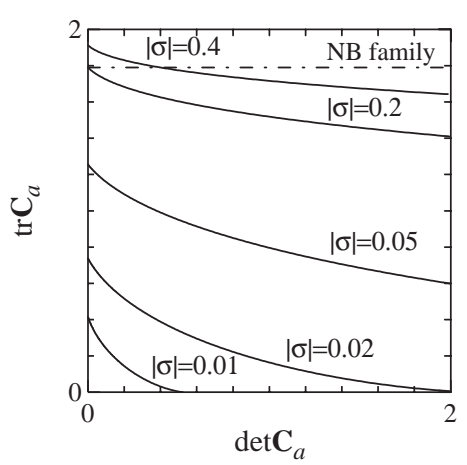

Fig. 12. $\mathscr{C}$ curves delimiting the unstable part of domain A for the NB cross section family $\left(\begin{array}{lll}c_{11} & c_{22} & 2.05\end{array}\right)$.

galloping oscillations of tower buildings. If the aerodynamic force coefficients reported in Ref. [6] are considered for varying angle of attack, a path belonging to domain $\mathrm{D}$ is obtained, a small part of which lies below the line $\mu_{\mathrm{cr}}^{0}=\mu_{D H}$. The conclusions of Ref. [6] are thus confirmed. Changing the expression of the lateral force coefficients in order to take into account the turbulence influence (e.g. [11]), the path now lies in A- and D-domains.

\subsection{Galloping motion}

The motion experienced by the system in conditions of incipient instability is now analyzed. The validity of the response is obviously confined to a small interval of time, since, when the amplitude of motion increases, the mechanical and aerodynamical nonlinearities, here neglected, decide on the nature of motion. Therefore, a nonlinear analysis, as that developed in Ref. [10], must be performed to investigate the system postcritical behaviour. Using the eigenvector expression (15), the projection of the trajectories on the $\left(q_{1}, q_{2}\right)$-configuration plane is evaluated. The motion subsequent to an initial horizontal displacement, $q_{1}=0.01, q_{2}=0$, for a wind velocity greater than the critical one, is analyzed.

The RMP example [4] is first considered, for which the representative point lies in domain D in the region $\mu_{\mathrm{cr}}^{0}<\mu_{D H}$. Figs. 13a c show the trajectories for three different values of detuning. When $\sigma=0$, since the point is outside the parabola $\operatorname{tr}^{2} \mathbf{C}_{a}-4 \operatorname{det} \mathbf{C}_{a}=0$ (see Fig. 4), eigenvectors (15) are real, i.e. motions develop in invariant subspaces of dimension 1 ; when $\sigma \neq 0$ the eigenvectors are complex, i.e. motions develop in invariant subspaces of dimension 2. Therefore, after an initial transient motion, the trajectories are attracted by the divergent mode, which is an inclined line for $\sigma=0$ (real mode of dimension 1, Fig. 13a), an elliptical spiral for small $|\sigma|$ (complex mode of dimension 2, Fig. 13b) and a flat nearly vertical spiral for large detuning (Fig. 13c), tending to the behavior of the single vertical dof. The amplitude of the elliptical spirals depend on the coupling between the horizontal and vertical motion through the term $d_{12}$, which is small for the considered case; the NB family too presents a similar behavior.

In order to obtain wider elliptical spirals, bimodal galloping cases need to be considered like those presented by NDT family in the domain B. Figs. 13d,e show the motion of the system $\mathrm{P}_{3}$ in Fig. 9, when $\sigma=0$ and $\sigma=0.02$, respectively. In resonance conditions, the corresponding 

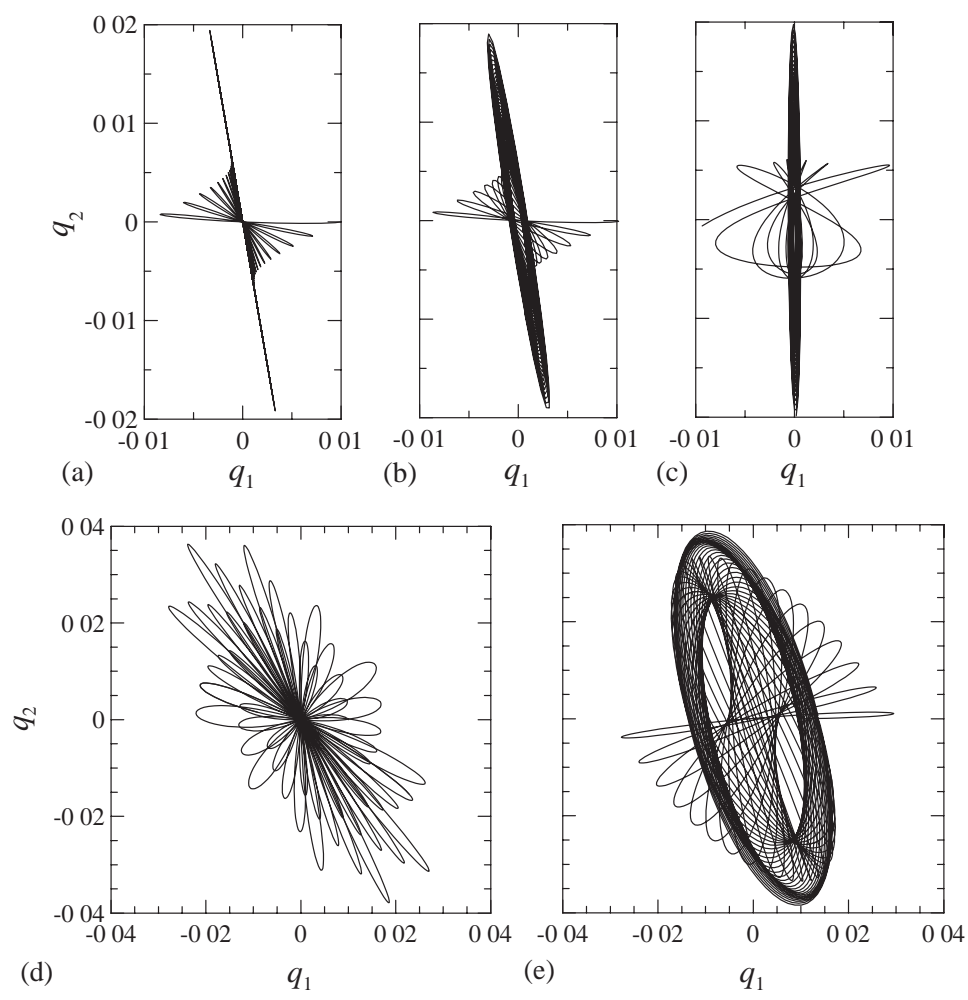

Fig. 13. Galloping motions: RMP cross section $\left(c_{d} \quad 1.07, c_{l} \quad 0.60, c_{d}^{\prime} \quad 1.06, c_{l}^{\prime} \quad 1.39\right)$, (a) $\sigma \quad 0, \mu_{\text {cr }} \quad 0.0266$, $\mu \quad 0.045$, (b) $\sigma \quad 0.02, \mu_{\mathrm{cr}} \quad 0.0287, \mu \quad 0.045$, (c) $\sigma \quad 0.5, \mu_{\mathrm{cr}} \quad 0.0435, \mu \quad 0.07$; NDT cross section (point $\mathrm{P}_{3}$ :

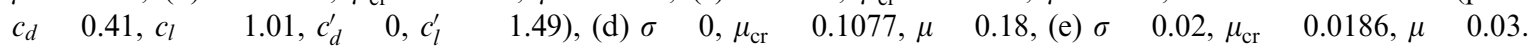

displacement time-histories presents a characteristic beat shape, called "complex galloping" in Ref. [4]. However, from the previous discussion on critical conditions, it is evident that the "complex galloping" feature is due to the occurring of a double Hopf bifurcation.

\section{Conclusions}

In this paper the bifurcation mechanisms of coupled flexural galloping, in resonant, quasiresonant and non-resonant conditions, have been analyzed by means of a perturbation approach, based on the ordering of mechanical and aerodynamic damping matrices. This method presents some differences with respect to the literature, where the exact analytical solutions are deduced in the resonant case only. The following conclusions are drawn.

1. The eigenvalue perturbative expressions are valid both in quasi-resonant and nonresonant regimes. Moreover, the quasi-resonant eigenvalues match excellently with the nonresonant one. 
2. From the perturbative eigenvalues, a closed-form for the critical velocity is derived. It coincides with the exact solution in the resonant case and presents good accordance with respect to the numerical solutions in quasi-resonant conditions.

3. The discussion of the eigenvalues in the plane of the invariants of the aerodynamic damping matrix permits a complete discussion of all the possible bifurcation mechanisms, both in resonant and quasi-resonant conditions.

4. In resonance and quasi-resonance conditions, Hopf bifurcations occur as (a) simple bifurcations (monomodal galloping), (b) quasi-simultaneous bifurcations (quasi-bimodal galloping) and (c) double bifurcations (bimodal galloping). Therefore, the true nature of the "complex galloping" identified in Ref. [4] is now highlighted: it occurs as a result of a double Hopf bifurcation instead of a classic Hopf bifurcation. The existence of an entire domain where it manifests itself is proved for resonant conditions.

5. The eigenvectors are real (i.e. the associated motion is mono-dimensional) if the detuning is equal to zero and the cross-section representative point is located outside the critical parabola in the $\operatorname{det} \mathbf{C}_{a}-\operatorname{tr} \mathbf{C}_{a}$ plane; in any other case they are complex (i.e. the motion is bidimensional). However, if the detuning is sufficiently large, the eigenvectors tend to real values (i.e. to vertical mono-dimensional motions).

6. In mono-modal galloping, the (initial) trajectories on the configuration plane are elliptical spirals, more or less flat depending on the smallness of the coupling term $d_{12}$. The vertical and horizontal amplitudes of motion are of comparable magnitude only for bimodal or quasibimodal galloping.

7. It is proved that cross-sections which are stable $\left(c_{22}>0\right)$ or unstable $\left(c_{22}<0\right)$ in a single vertical dof schematization, possibly become unstable or stable, respectively, if the horizontal dof is introduced in the model. Moreover, a section which is stable in resonance conditions may become unstable at a sufficiently large detuning $\sigma$, although it regains stability at high wind velocity (transient instability phenomenon).

8. The perturbative results can easily be used for technical purposes. From the position of the representative point in the plane it is immediately known whether the resonant critical of the invariants velocity is above or below the Den Hartog value (Fig. 5). Then, the qualitative pattern of the critical velocity vs. detuning curve is drawn.

\section{Acknowledgements}

This study has been partially supported by the Italian Ministry of University (MIUR) through a co-financed program.

\section{Appendix A. Perturbation analysis of the eigenvalue problem}

The computational aspects of the perturbation analysis performed in Section 3 are illustrated here. Since the original problem (5) is homogenous, a suitable normalization condition must be enforced for the eigenvector $\mathbf{w}$. The condition $\mathbf{B w}=1$ is chosen, where the operator $\mathbf{B}$ extracts one component of $\mathbf{w}$. Consequently, $\mathbf{B} \mathbf{w}_{0}=1$ and $\mathbf{B} \mathbf{w}_{1}=0$ are normalization conditions for the 
$\varepsilon^{0}$ - and $\varepsilon$-order perturbation equations (7) and (11). The non-resonant $(|\omega-1|>O(\varepsilon))$ and quasiresonant $(|\omega-1|=O(\varepsilon))$ cases are dealt with separately.

\section{A.1. Non-resonant case}

The perturbation equations (7) must be solved. Eq. (7a) is the eigenvalue problem of the undamped system, whose solution reads:

$$
\varepsilon^{0}: \lambda_{0}^{(1,2)}= \pm \mathrm{i} \omega, \quad \mathbf{w}_{0}^{(1,2)}=\left\{\begin{array}{l}
1 \\
0
\end{array}\right\}, \quad \lambda_{0}^{(3,4)}= \pm \mathrm{i}, \quad \mathbf{w}_{0}^{(3,4)}=\left\{\begin{array}{l}
0 \\
1
\end{array}\right\} .
$$

By substituting these solutions in the $\varepsilon$-order equation (7b) and requiring the right-hand member to be orthogonal to the eigenvector $\mathbf{w}_{0}$ (solvability condition), $\lambda_{1}=-\mathbf{w}_{0}^{\mathrm{T}} \mathbf{D} \mathbf{w}_{0} / 2$ follows, i.e.:

$$
\lambda_{1}^{(1,2)}=-\frac{1}{2} d_{11}, \quad \lambda_{1}^{(3,4)}=-\frac{1}{2} d_{22} .
$$

By solving Eq. (7b) with the relevant normalization condition and by substituting the results obtained in Eqs. (6), Eqs. (8) follows, where the parameter $\varepsilon$ has been reabsorbed.

\section{A.2. Resonant and quasi-resonant cases}

The relevant perturbation equations are Eqs. (11). Since $\lambda_{0}^{(1,2)}=i, \lambda_{0}^{(3,4)}=-i$ are eigenvalues of double multiplicity for Eq. (11a), the associated eigenvectors are indeterminate, namely:

$$
\mathbf{w}_{0}=\sum_{i=1}^{2} \alpha_{i} \mathbf{u}_{i}, \quad \mathbf{u}_{1}=\left\{\begin{array}{l}
1 \\
0
\end{array}\right\}, \quad \mathbf{u}_{2}=\left\{\begin{array}{l}
0 \\
1
\end{array}\right\},
$$

where $\alpha_{i}$ are arbitrary constants. In order that Eq. (11b) admits solution, its right-hand term must be orthogonal to each of $u_{i}$ [12], that is:

$$
\sum_{i=1}^{2}\left(2 \lambda_{0} \lambda_{1} \mathbf{u}_{j}^{\mathrm{T}} \mathbf{u}_{i}+\mathbf{u}_{j}^{\mathrm{T}} \mathbf{K}_{1} \mathbf{u}_{i}+\lambda_{0} \mathbf{u}_{j}^{\mathrm{T}} \mathbf{D}_{0} \mathbf{u}_{i}\right) \alpha_{i}=0, \quad j=1,2 .
$$

Eq. (A.4) represents a new eigenvalue problem. By setting its determinant to zero, two generally different corrections $\lambda_{1}^{(k)}$ of each eigenvalue $\lambda_{0}^{(k)}$ are obtained, i.e. the $\varepsilon$-order perturbation destroy the degeneracy. Finally, by substituting $\lambda_{1}=\lambda_{1}^{(k)}$ into Eqs. (A.4) and using the normalization condition, the coefficients $\alpha_{i}$ are determined. Thus, by reabsorbing the parameter $\varepsilon$, the following approximate eigensolutions are obtained:

$$
\begin{aligned}
& \lambda^{(1,2)}=\mathrm{i}-\frac{d_{11}^{0}+d_{22}^{0}}{4}+\frac{\mathrm{i} \sigma}{2} \pm \frac{1}{4} \sqrt{ }\left(d_{11}^{0}-d_{22}^{0}\right)^{2}+4 d_{12}^{0} d_{21}^{0}-4 \mathrm{i} \sigma\left(d_{11}^{0}-d_{22}^{0}\right)-4 \sigma^{2}, \\
& \lambda^{(3,4)}=-\mathrm{i}-\frac{d_{11}^{0}+d_{22}^{0}}{4}-\frac{\mathrm{i} \sigma}{2} \pm \frac{1}{4} \sqrt{ }\left(d_{11}^{0}-d_{22}^{0}\right)^{2}+4 d_{12}^{0} d_{21}^{0}+4 \mathrm{i} \sigma\left(d_{11}^{0}-d_{22}^{0}\right)-4 \sigma^{2}, \\
& \mathbf{w}^{(k)}=\left(-\frac{d_{12}^{0} \lambda_{0}^{(k)}}{2 \lambda_{0}^{(k)} \lambda_{1}^{(k)}+2 \sigma+\lambda_{0}^{(k)} d_{11}^{0}}, \quad 1\right)^{\mathrm{T}}, \quad k=1, \ldots, 4,
\end{aligned}
$$




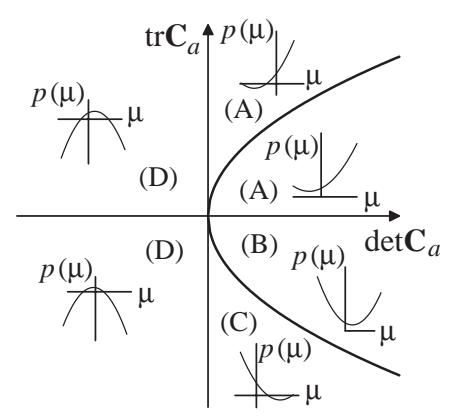

Fig. 14. Discussion of the roots of the polynomial $p(\mu)$.

namely, Eqs. (12) (15). It should be noted that, due to the indeterminacy of the generating solution (A.3), the $\varepsilon$-order correction $\mathbf{w}_{1}$ of the eigenvector can be evaluated only at the $\varepsilon^{2}$-order.

\section{Appendix B. Study of the function $g(\mu)$}

The graph of the function $g(\mu)$ appearing in Eq. (24) is studied for $\mu \geqslant 0$. It is:

$$
g(0)=0, \quad \lim _{\mu \rightarrow \infty} g(\mu)=\frac{1}{\sqrt{ } \operatorname{det} \mathbf{C}_{a}},\left.\quad \frac{\mathrm{d} g(\mu)}{\mathrm{d} \mu}\right|_{\mu=0}=\frac{1}{\sqrt{4 \xi_{s}^{2}+\sigma^{2}}}, \quad \mu_{0}=-\frac{4 \xi_{s}^{2}+\sigma^{2}}{\xi_{s} \operatorname{tr} \mathbf{C}_{a}},
$$

$\mu_{0}$ being the point of stationariness. In order that $\mu_{0}>0, \operatorname{tr} \mathbf{C}_{a}<0$; therefore $g(\mu)$ is monotone if $\operatorname{tr} \mathbf{C}_{a}$ is positive, whereas it has a unique steady-point if $\operatorname{tr} \mathbf{C}_{a}$ is negative. Moreover, the function $g(\mu)$ admits vertical asymptotes at the zeros of the polynomial $p(\mu)$ in Eq. (23c). It is easy to verify that the roots of $p(\mu)$ are real outside the parabola $\operatorname{tr} \mathbf{C}_{a}^{2}-\left(4+\sigma^{2} / \xi_{s}^{2}\right) \operatorname{det} \mathbf{C}_{a}=0$ and complex inside it. Moreover, only the positive roots are of interest. The results of the complete discussion of the equation $p(\mu)=0$ are summarized in Fig. 14. From it, the patterns of $g(\mu)$ represented in Fig. 8 follow. In fact, in both domains A and B, the denominator of $g(\mu)$ is different from zero, thus no asymptotes exist. Moreover, in domain A, $\mu_{0}$ is negative, so no point of stationariness occurs; instead, in B there is one point of stationariness. With regard to the C- and D-domains, it is possible to prove that the point of stationariness is located in the interval of non-existence of $g(\mu)$.

\section{References}

[1] G. Solari, Gust excited vibrations, in: H. Sockel (Ed.), Wind Excited Vibrations of Structures, Springer, Vienna, Austria, 1994, pp. 195291.

[2] G. Piccardo, Analysis of Coupled Aeroelastic Phenomena, PhD Thesis, University of Florence, Italy, 1993 (in Italian).

[3] Y.M. Desai, A.H. Shah, N. Popplewell, Galloping analysis for two degree of freedom oscillator, Journal of Engineering Mechanics 116 (12) (1990) 25832602.

[4] K.F. Jones, Coupled vertical and horizontal galloping, Journal of Engineering Mechanics 118 (1) (1992) 92107. 
[5] P. Yu, N. Popplewell, A.H. Shah, Instability trends of inertially coupled galloping, Part I: initiation, Journal of Sound and Vibration 183 (4) (1995) 663-678.

[6] L. Shuguo, L. Qiusheng, L. Guiqing, Q. Weilian, An evaluation of onset wind velocity for 2-D coupled galloping oscillations of tower buildings, Journal of Wind Engineering and Industrial Aerodynamics 50 (1993) 329-340.

[7] G. Piccardo, A. Luongo, Stability critical conditions for coupled flexural galloping, Proceedings of the XII Italian Conference of Theoretical Applied Mechanics, Vol. II, Naples, Italy, October 1995, pp. 161-166 (in Italian).

[8] G. Piccardo, A methodology for the study of coupled aeroelastic phenomena, Journal of Wind Engineering and Industrial Aerodynamics 48 (1993) 241-252.

[9] R.D. Blevins, Flow-induced Vibration, second ed., Van Nostrand Reinhold, New York, 1990.

[10] A. Luongo, A. Paolone, G. Piccardo, Postcritical behavior of cables undergoing two simultaneous galloping modes, Meccanica 33 (1998) 229-242.

[11] P.W. Bearman, I.S. Gartshore, D.J. Maull, G.V. Parkinson, Experiments on flow-induced vibration of a squaresection cylinder, Journal of Fluids and Structures 1 (1987) 19-34.

[12] A. Luongo, Eigensolutions sensitivity for nonsymmetric matrices with repeated eigenvalues, AIAA Journal 31 (7) (1994) 1321-1328. 